\author{
4. Teil: \\ Organisatorische Ausgestaltungen justizieller Selbstverwaltung: \\ Auswahl, Beurteilung und Beförderung
}

\author{
Giovanni Salvi
}

\title{
Das italienische System »offener Rollen«: Beurteilungen im Rahmen autonomer Laufbahnstrukturen einer selbstverwalteten Justiz
}

\section{Justizielle Beurteilungspraxis}

Die wichtigste, unverzichtbare Bastion bei der Verteidigung der Autonomie und Unabhängigkeit der Richter und Staatsanwälte ${ }^{1}$ ist diejenige solider professioneller Fertigkeiten. $^{2}$

\section{Auswahl der Richter und Staatsanwälte}

Der erste Schritt zur Sicherstellung eines hohen Niveaus von Professionalität ist der Auswahlprozess. Jede Auswahl ist stark beeinflusst von Angebot und Nachfrage, die wiederum von Faktoren abhängen, welche sich gänzlich außerhalb der Kontrolle des Obersten Justizrates (»Consiglio Superiore della Magistratura $«=C S M$ ) befinden. Dazu gehören beispielsweise die Vergütung im Vergleich mit ähnlichen Beschäftigungen oder die Wahrnehmung des mit dem Amt verbundenen Prestiges durch die Bevölkerung. In Italien gilt es ebenfalls die starke Favorisierung einer unkündbaren Beschäftigung im öffentlichen Dienst zu bedenken, die als die typische Ressource von Aufsteigern aus südlichen Landesteilen gesehen wird. Gegenwärtig erfolgt die Zulassung durch öffentliche Prüfung, teilweise mittels einer Qualifikation zweiten Grades (frühere juristische Erfahrung oder passender Masterabschluss) und teilweise durch Vorauswahl. Die öffentliche Prüfung besteht aus mündlichen und schriftlichen Teilen.

1 Richter und Staatsanwälte gemeinsam werden im italienischen Rechtssystem als Magistrati bezeichnet. Wenn im Folgenden die Bezeichnung Richter allein verwendet wird, sind auch Staatsanwälte einbezogen.

2 Der CSM verwendet die benutzten Ausdrücke in folgender Weise: »Unabhängigkeit ist zu verstehen als die Ausübung richterlicher Funktionen ohne Vorbedingungen, Beziehungen oder Verbindungen, die die Art der Ausübung richterlicher Tätigkeit limitieren oder nachteilig beeinflussen könnten. Unparteilichkeit zeigt sich in einem fairen Verhalten seitens der Richter gegenüber den im Verfahren beteiligten Parteien. Schließlich setzt sich Ausgeglichenheit zusammen aus der Erledigung von Pflichten mit Mäßigung und Sinn für Verhältnismäßigkeit, frei von ideologischer, politischer oder religiöser Befangenheit. Diese Bedingungen stellen die notwendige Voraussetzung für ein positives richterliches Beurteilungswesen dar« (Rundschreiben Nr. 20691, 8. Oktober 2007). 
Nach einer zweijährigen Ausbildungszeit werden die Kandidaten zum ersten Mal beurteilt und es werden ihnen spezifische rechtsprechende Funktionen zugewiesen.

2. Probleme der Beurteilung justizieller »Professionalität «: geschichtlicher Rückblick und Reformbedarf

a) Probleme des Beurteilungswesens

Die laufbahnmäßige Beförderung ist abhängig von regelmäßigen Bewertungen, die in der Verantwortung des CSM liegen und die auf Grundlage der von den Gerichtspräsidenten, Leitern der Staatsanwaltschaften und den Justizräten (Consigli Giudiziari) erstellten Beiträge erfolgen. Diese Beurteilungen richterlicher und staatsanwaltlicher »Professionalität « sind der italienischen Justiz zwar ein Dorn im Auge, gleichwohl beinhaltet diese Praxis ein System gegenseitiger Kontrolle, welches verhindert, dass über das Instrument professioneller Laufbahnbeurteilungen der Inhalt richterlicher und staatsanwaltlicher Entscheidungen und ideologische Tendenzen beeinflusst werden.

aa) Inhaltsleere Beurteilungspraxis

Lange hat der Versuch, Autonomie und Unabhängigkeit der Justiz zu sichern, mit automatischer Beförderung und automatischen Beurteilungen geendet, die immer gleich und damit inhaltsleer waren. Viel zu oft haben die Richtervereinigungen lediglich Lippenbekenntnisse bezüglich eines Konzeptes abgelegt, welches im justizeigenen richterlichen Beurteilungswesen eine zentrale Garantie der Unabhängigkeit der Dritten Gewalt, aber auch ein berechtigtes Interesse der Bürger an einer funktionierenden Justiz sieht. Der Oberste Justizrat wiederum hat zu spät reagiert und Berichte toleriert, die in möglichst allgemeiner Form abgefasst waren und keinen direkten Bezug zur Tätigkeit der Richter und Staatsanwälte hatten. Wir haben in einer unglaubwürdigen Welt gelebt, in der jeder als »exzellent « beurteilt wurde und - in stilistisch vollendeter Form - Komplimente für sein »vorzügliches Verhalten« bekam.

bb) Irrweg politischer Maßnahmen

Andererseits haben sich auch politische Maßnahmen als ineffektiv erwiesen. Die Bedrohung durch Bewertungsverfahren ist benutzt worden, um die Justiz einzuschüchtern, anstatt sich ernsthaft der zahlreichen Probleme anzunehmen, welche bei der Bewertung richterlicher und staatsanwaltlicher Arbeit im Sinne einer verfassungsmäßig geschützten Berufsrolle entstehen. Die Reformgesetze für Justiz und Recht aus dem Jahr 2006 gingen diese Fragen in weiten Teilen unangemessen an. Sie intendierten die Rückkehr zu einem System der internen Revision, das in den 50er und 60er Jahren schon existierte, sich aber bereits als unbefriedigend erwiesen hatte. Um zu einem genaueren Verständnis der schwierigen Balance dieser Interessen zu kommen, ist es unabdingbar, die Verbindung zwischen dem Beurteilungswesen und der Karriere der Richter und Staatsanwälte zu betrachten.

b) Geschichtliche Entwicklung der richterlichen Laufbahnstruktur

Anfang der 70er Jahre gab es zwei bedeutende Änderungen in der Laufbahnstruktur der Richter und Staatsanwälte. Einerseits wurde der Zusammenhang zwischen der hie- 
rarchischen Dienststellung und der Besoldung aufgehoben. Auch wenn ein Richter bzw. Staatsanwalt stets an Untergerichten eingesetzt war, konnte er nun die höchste Qualifikationsstufe, die Richter und Staatsanwälte überhaupt erreichen können (»wählbar für hohe leitende Funktionen«), einnehmen. Andererseits wurde das Verbot amtlicher Zurückstufung aufgehoben. Dies hatte zur Folge, dass ein Richter oder Staatsanwalt von den höheren Gerichten und Staatsanwaltschaften (sogar vom Kassationsgericht) auf Antrag wieder zu einer niedrigeren Gerichtsbarkeit versetzt werden konnte. Diese beiden Reformen zielten unter verfassungsrechtlichen Aspekten auf etwas außerordentlich Wichtiges: Auf die Einführung der verfassungsmäßigen Vorkehrungen, gemäß derer einzelne Richter bzw. Staatsanwalt lediglich aufgrund der von ihnen ausgeübten Funktionen voneinander unterschieden werden können (Art. 107 der Verfassung). Dies war verbunden mit dem verfassungsmäßigen Konzept von Rechtsprechung als einer diffundierten Gewalt, d.h. einer Gewalt, die die Richter- und Staatsanwaltschaft durchdringt und nicht eine lediglich von oben delegierte Gewalt ist (potere diffuso). Dadurch wollte man das ehrgeizige Ziel erreichen, Richter auch innerhalb der Dritten Gewalt effektiv, autonom und unabhängig zu machen. So sollte sichergestellt werden, dass die Richter Recht sprechen können, ohne befürchten zu müssen, dass ihre Karriere durch äußere Anreize - wie finanzielle Vorteile oder Aufstiegseinschränkungen - beeinflusst werden könnte. Sie sollten frei sein von äußeren und inneren Einflüssen jeglicher Art. Diese Innovationen gingen Hand in Hand mit der Abschaffung der internen Revisionen, die es höheren Richtern und Staatsanwälten bis dahin ermöglicht hatten, eine strenge Kontrolle über untergeordnete Richter bzw. Staatsanwälte auszuüben.

Ein weiterer Faktor, der das Reformprogramm der 70er Jahre antrieb, war die zunehmende Unzufriedenheit der Öffentlichkeit mit der Art, in der sich die Justiz - anscheinend instinktiv - vorherrschenden politischen Trends anzupassen verstand. Die Justiz wurde verdächtigt, reaktionäre und gänzlich politisierte Positionen unter dem Deckmantel der Neutralität zu verbergen. Sie wurde auch als unfähig angesehen, dem Gerechtigkeitsbedürfnis einer Gesellschaft zu entsprechen, die sich als komplex, dynamisch und »offen« versteht. Insofern nahm sich der CSM gezielt aller internen Arbeitsvorgänge der Justiz an, wie es in der Verfassung vorgesehen ist. Dies führte zu einer substantiellen Steigerung der Unabhängigkeit der Justiz, nachweislich nicht nur im strafrechtlichen Sektor (durch die Einführung von Strafgesetzen für Zusammenhänge, die bis dahin dagegen immun gewesen waren), sondern auch im zivilrechtlichen Bereich. Hier wuchs der Justiz eine zunehmend bedeutendere Rolle bei der Sicherstellung von Rechten in Rechtsfeldern zu, die zuvor vernachlässigt worden waren, wie zum Beispiel im Arbeits-, Umwelt- und Persönlichkeitsrecht.

Im Gegenzug führte die unabhängigere Rolle der Justiz dazu, dass sie immer mehr in Streitigkeiten mit dem politischen System verwickelt wurde. Das Bemühen der Justiz, sich erstmals intensiv mit Terrorismus, organisiertem Verbrechen und Korruption zu beschäftigen, stellte einen Wendepunkt dar. Aber auch heute ist die Justiz nur in sehr geringem Maße für Funktionsausfälle verantwortlich. Schon die hohe Anzahl an Berufungsverfahren am Obersten Gericht zeigt, wie dysfunktional die Justiz aufgestellt ist: $2007=32.278$ Berufungsverfahren im Zivil- und 43.732 im Strafrechtsbe- 
reich. Ganze fünf Richter und ein Vertreter der Generalstaatsanwaltschaft sind gezwungen, sich entweder mit zivilen Streitigkeiten geringen Streitwertes ohne jedes öffentliche Interesse oder mit zeitverzögernden Anträgen in Strafverfahren zu beschäftigen.

Zusammenfassend bleibt der Befund, dass das richterliche Beurteilungswesen eine lange Zeit Gefahr lief, zu einem »gelangweilten Ritual« zu denaturieren. Die zentralen Prinzipien, die der Reform der 70er Jahre zugrundelagen, drohten in den Fängen des Korporatismus zu ersticken.

c) Das Beurteilungssystem in Italien in Abgrenzung zu anderen europäischen Modellen

Das Beurteilungssystem, das wir in Italien kennen, kann nicht als Vorbild für andere Länder dienen. Es ist fest verbunden mit einem Modell der Justiz, in dem Richter/ Staatsanwälte Beamte sind, die eine eigene Körperschaft bilden. Sie werden rekrutiert durch öffentliche Prüfung, sind aufgewachsen mit traditionellen Karrierevorstellungen und werden befördert mittels einer regelmäßigen, spezifischen Art der Bewertung. Der Mechanismus der Bewertung begleitet den Richter und Staatsanwalt im Verlauf seiner Karriere und soll überprüfen, dass er oder sie eine Beförderung verdient. Das Beurteilungssystem entscheidet hauptsächlich nach der sich aus schriftlichen Berichten ergebenden Aktenlage; es basiert auf einer Logik des Schutzes des Richters und Staatsanwalts vor Fehleinschätzungen, Missbrauch oder äußerem Druck. Die Beurteilungen werden durch die Dritte Gewalt selbstverantwortlich erstellt, unterliegen aber letztlich der Verwaltungsrechtsprechung. Dies ist ein Modell, das aus der französischen Erfahrung entstanden ist (wenn auch mit zahlreichen Unterschieden) und sich grundlegend unterscheidet von dem der »Common-Law «-Länder und von den Ländern Nordeuropas, wenigstens was die Methoden und Ziele der Beurteilung betrifft. Gleichwohl wäre es falsch anzunehmen, dass kontinental-europäische Länder justizielle Leistungsbeurteilungen völlig außer Acht lassen. Wie auch immer: Für alle Beurteilungssysteme sollte beachtet werden, dass die beiden Verfahren der Messung und Bewertung voneinander gänzlich unabhängig sein müssen, sowohl was das Ziel als auch die Methode betrifft.

d) Reaktionen des Obersten Justizrates (CSM)

Die Aufgaben des CSM sind beschrieben im Bericht über seine verfassungsmäßige Rolle und seine Beziehungen zum Justizminister, der für die Organisation der Justizdienste verantwortlich ist. Der CSM reagierte auf die bisherige Schwäche der Bewertungssysteme in mehrfacher Hinsicht.

\section{aa) Zusammenarbeit mit dem Justizministerium}

Erstens begann der CSM die Zusammenarbeit mit dem Justizministerium in statistischen Angelegenheiten aufzunehmen. Ein integriertes System von gleichartigen Indikatoren der Beurteilung und des Vergleichs der Effizienz verschiedener Justizbehörden wurde erarbeitet. Standards wurden erstellt, um eine vergleichende statistische Bewertung der Arbeit des Richters bzw. Staatsanwalts zu ermöglichen (Erlass des 
CSM Nr. 20691/07). Solche Standards werden gegenwärtig in unterschiedlichen Regionen erprobt.

bb) Einbeziehung der richterlichen Entscheidung in die Bewertung

Zweitens führte er gleichzeitig eine neue Bewertungspraxis von Richtern und Staatsanwälten ein, die es weder in den kontinentalen noch in den nordeuropäischen Systemen gibt: Die Bewertung hatte die Analyse hinsichtlich einzelner, vom Richter bzw. Staatsanwalt erstellter Entscheidungen zu erfassen. Es wird sofort klar, dass dies ein äußerst heikler Schritt ist, der nur in einem Land wie Italien verständlich ist, welches eine lange und verworrene Entwicklung seines Justizsystems erfahren hat. Die Idee, dass man die Rechtsprechung von Richtern bewerten kann, wäre sicherlich unannehmbar für die Justizsysteme zum Beispiel in Frankreich, Finnland, Dänemark oder Portugal. Sie würden darin ein System der unzulässigen Kontrolle der Urteile und ihres Inhalts sowie eine unzulässige Beeinträchtigung der Autonomie des Richters sehen. Es ist jedenfalls keine leichte Aufgabe, Zustimmung dafür zu finden, dass die Überprüfung von Maßnahmen nicht zwangsweise den Inhalt der Entscheidung berühren muss und dass es - dank der Tatsache, dass mit der Kontrolle ausschließlich der CSM betraut ist - möglich ist, Beurteilungen vorzunehmen, die nicht als Vehikel missbraucht werden, die Freiheit und Unabhängigkeit des Richters einzuschränken.

cc) Standardisierung von Bewertungskriterien

Die Hauptrichtung der Reforminitiativen des CSM hat sich auf qualitative Kriterien konzentriert, die der justiziellen Beurteilung zugrunde zu legen sind. Deswegen war in der autonomen Justizverwaltung sicherzustellen, dass die von dort kommenden Beurteilungsgrundlagen transparenter und überzeugender wurden (Rundschreiben $\mathrm{Nr}$. 16103, 30. Juli 2003). Eine Schlüsselrolle spielt die Vorgabe eines standardisierten, homogenen Rasters zur Sammlung der Auffassungen der Justizräte sowie der autonomen Justizverwaltung. Was wirklich neu ist an der Einführung eines Rasters für die Erstellung von Bewertungen ist erstens, dass es anstelle generalisierter, stereotyper Stellungnahmen die Integration von Fakten vorschreibt, und es zweitens darauf abzielt, die Bewertungen der unterschiedlichen Justizräte zu standardisieren.

dd) Einführung der Selbstbeurteilung

Zudem war die Einführung eines persönlichen Berichts als Selbstbeurteilung vorgesehen, um dem zu bewertenden Richter bzw. Staatsanwalt mehr Verantwortung für seine eigene Leistung zu geben. Der persönliche Selbstbericht ist einer der Schlüsselkomponenten bei der Befähigung der Justizräte, sich eine Meinung zu bilden. Er liefert Fakten als Beurteilungskriterien. Der Rückgriff auf Arbeitsmuster justizieller Tätigkeit für Bewertungszwecke ist ebenso in hohem Maße innovativ.

e) Risiken für die richterliche Unabhängigkeit durch die Einführung von standardisierten Bewertungen

Die Beurteilung der Professionalität eines Richters beinhaltet ein Risiko bezüglich seiner Unabhängigkeit. Je detaillierter die Bewertung ist, umso größer ist auch die Gefahr, dass sie durch die Unterwerfung seiner Aktivitäten unter Bewertungsurteile die 
Grenzen richterlicher Autonomie überschreitet. Es ist unmöglich, diese Spannung zwischen beruflicher Beurteilung und Unabhängigkeit aufzuheben, aber es ist wichtig, sich dieser Spannungen bewusst zu bleiben.

Das bestimmende Kriterium für die Sicherstellung, dass professionelle Beurteilungen nicht in Einmischungen in die Rechtsprechung umschlagen, wird durch die Grenzen ausgedrückt, die jeder Bewertung gesetzt sind: Die Bewertung ist zu beschränken »auf ausschließlich technische und professionelle Aspekte, die die Auslegung der Fragen und Argumente zur Unterstützung der getroffenen Entscheidung betreffen, aber unter Ausschluss jedweder Beurteilung zum Beispiel des Wertes der Entscheidung selbst $\ll$.

Ein weiteres Risiko im Hinblick auf die Einführung von formalen Vorgaben besteht darin, dass sie eine gewisse Konformität richterlicher Arbeitsweisen erzeugen könnten. Dieser Konformitätsdruck dürfte dazu führen, eine »perfekte« Maßnahme treffen zu wollen, wie sie in den standardisierten Vorgaben als »nützlich« dargestellt werden. Dabei darf die »Perfektion« einer Entscheidung nicht abhängig sein von strikter Ausrichtung an rechtsdogmatischen Kategorien und höherer Rechtsprechung, sondern muss sich an für richterliche Arbeitsweisen geschaffenen Struktur- und Motivationsvorgaben orientieren. Im Ergebnis: Formelle Bewertungsvorgaben müssen gleichwohl offen sein für sich ständig wandelnde Anforderungen.

\section{Das System der »offenen Rollen«}

Ein besonderes Problem in der Vergangenheit war die Wiederholung von Beurteilungen in sehr langen Intervallen. Zum Beispiel war eine erneute Beurteilung eines Richters bzw. Staatsanwalts, der einmal für ein niedrigeres Gericht eingestuft wurde, erst wieder nach zehn Jahren vorgesehen. Eine andere Beurteilung war in dieser Periode nicht zugelassen, die gesetzlichen Vorschriften schlossen jede andere Möglichkeit aus. In der Praxis war eine ganze Reihe von Maßnahmen erforderlich, um eine solche Untätigkeit auszugleichen.

Die Justizreform von 2005/2006 hat den Referenzrahmen radikal verändert, indem sie schließlich Beurteilungen in kürzeren Zeitabständen vorschrieb und Qualifikationen nicht länger mit beruflichem Aufstieg in höhere Positionen verband. ${ }^{3}$ Weiterhin hat die Reform eine feste Frist für die Dauer von Berufungen in Leitungsfunktionen (Gerichtspräsident/Leiter der Staatsanwaltschaft; 4 Jahre, nur einmal erneuerbar) möglich gemacht.

Wenn dieses Maßnahmenpaket - wie erwartet - Früchte trägt, wird es die aufgezeigten negativen Aspekte des früheren Systems, die oben beschrieben wurden, besei-

3 Die Reform von 2005/2006 (bestätigt durch die gesetzlichen Regelungen von 2007) setzte die folgenden Positionen außer Kraft: Uditore giudizario (nicht identisch, aber vergleichbar mit »Proberichtern «) mit oder ohne Funktionen, Amtsrichter, Richter/Staatsanwalt beim Appellationsgericht und beim Kassationsgericht, leitende Positionen. Diese Posten wurden ersetzt durch eine Skala von sieben Altersstufen, jede mit einer Dauer von vier Jahren und korrespondierend mit der Eignung, spezifische Funktionen $\mathrm{zu}$ übernehmen, und der Berechtigung auf die entsprechende Regel-Gehaltseinstufung. 
tigen. Das System der »offenen Rollen« hat sich als äußerst erfolgreich erwiesen bei der Verbesserung der inneren Autonomie der Richter und Staatsanwälte und - in der Folge - auch bei der Sicherstellung, dass die Justiz wirklich (und nicht nur dem Wortlaut nach) eine »diffundierte « und nicht hierarchisierte Gewalt ist.

Gleichzeitig zeigt das System gravierende Mängel bei der Bereitstellung angemessener Bewertungsmaßstäbe für berufliche Begabungen und Talente, die den Einzelnen für die ausgeübte Tätigkeit besonders qualifizieren. Konsequenterweise hat der Oberste Justizrat das oben beschriebene Beurteilungssystem übernommen, um den neuen Vorschriften zu entsprechen ${ }^{4}$, ohne jedoch seine grundlegende Ausrichtung zu ändern. Wie bereits gezeigt wurde, hat der Oberste Justizrat versucht, die Untätigkeit der Legislative durch Aufstellung sekundärer Normen ${ }^{5}$ auszugleichen. Die Legislative hat ihrerseits später das vom CSM geschaffene System übernommen, indem bei Beurteilungen »objektive Indikatoren« verwendet werden. Bemerkenswert ist, dass im Rahmen dieses neuen Bewertungssystems den Informationen größere Bedeutung zukommt, die von den neuerdings in den Justizräten als Mitglied wirkenden Rechtsanwälten angeboten werden. Die größte Neuerung sind Beurteilungen nach jeweils vier Jahren. Wie in Art. 11, Gesetz Nr. 111/2007 nun vorgesehen, kann das Beurteilungsverfahren mit drei möglichen Urteilssprüchen des Obersten Justizrats enden:

(a) Positives Ergebnis, d.h. das Ergebnis ist positiv, wenn die Beurteilung bezüglich aller Parameter der Untersuchung positiv ausfällt. In diesem Falle erhält der Richter

4 Rundschreiben Nr. 20691, 8. Oktober 2007.

5 In Anerkennung der Formulierungen des CSM legt Art. 11 des Gesetzes 111/2007 die Parameter der Beurteilungskriterien fest. Sie sind wie folgt im Rundschreiben des CSM beschrieben: »Die Leistungsfähigkeit (capacity) hängt zusammen mit rechtlichem Wissen und der Annäherung an gegenwärtige, normative, lehrmäßige und rechtsprechende Maßnahmen; einem sicheren Verständnis der Argumentations- und Befragungstechniken, ebenso im Verhältnis zum Resultat der nachfolgenden Abschnitte des Verfahrens; von der Art der Verfahrensleitung seitens der Vorsitzenden, von der Kompetenz der Benutzung, der Leitung und Kontrolle der Beiträge von Mitarbeitern und Gehilfen; von einer Fähigkeit angemessen mit anderen Dienststellen mit ähnlichem Aufgabenbereich zusammenzuarbeiten. Wirtschaftlichkeit hängt ab von Produktivität, verstanden als die Anzahl und Qualität von bearbeiteten Fällen in Relation mit dem Typ, der Organisation und der Behördenstruktur; der benötigten Zeit für die Abarbeitung der vorhandenen Aufgaben; Belege der Zusammenarbeit innerhalb des Gerichts. Gewissenhaftigkeit und Sorgfalt hängt ab von Eifer und Pünktlichkeit in Bezug auf den Dienstantritt und Anhörungen zu den angesetzten Tagen; der Beachtung von Zeitvorgaben für Registrierungsmaßnahmen und die Vervollständigung von gerichtlichen Pflichten; die Teilnahme an von der Justizverwaltung disponierten Sitzungen zum Zweck der Diskussion und Analyse neuer legislativer Maßnahmen, genauso wie für die Erweiterung des Wissens über Rechtsprechung; Verantwortlichkeit hängt ab von der Verfügbarkeit, abwesende Kollegen zu ersetzen mit dem Ziel einen reibungslosen Geschäftsablauf zu garantieren, sowie der Einhaltung der rechtlichen Normen und den Direktiven des CSM; der Bereitschaft zur regelmäßigen Teilnahme an Fortbildungskursen, die von der Höheren Juristenschule (»Higher Judiciary School«) organisiert werden oder (im Hinblick auf die Tatsache, dass Zugang zu diesen Kursen nicht allein von der Bewerbung des Richters abhängt) mit der Maßgabe zu akzeptieren, dass die relevanten Kurse bis zum Zeitpunkt, zu dem die oben genannte Schule funktionsfähig ist, durch den CSM organisiert werden; die Assistenz bei der Problemlösung von organisatorischen und juristischen Fragen mit dem Ziel, hastige, ergebnislose und unkoordinierte Initiativen zu vermeiden «. 
bzw. Staatsanwalt die volle Auszeichnung von Professionalität mit der gesetzlich vorgesehenen Regel-Gehaltserhöhung und der Befugnis, andere Funktionen zu übernehmen.

(b) Kein positives Ergebnis, d.h. es wurden Unzulänglichkeiten festgestellt. In diesem Fall führt der CSM weitere Bewertungen nach einem Jahr durch und verlangt eine neue Beurteilung vom Justizrat.

(c) Negatives Ergebnis: Im Falle ernstlicher Verfehlungen kann das Ergebnis variieren zwischen verpflichtender Nachschulung bis zum Ausschluss von bestimmten Pflichten oder leitenden Positionen und bis zur Entlassung aus dem Dienst. Die Entlassung ist möglich nach einer weiteren zweijährigen Versuchsperiode, der eine zweite negative Beurteilung folgt. Das Ergebnis des Beurteilungsverfahrens unterliegt natürlich gegebenenfalls den für ein streitiges Verfahren maßgeblichen Verfahrensregeln.

\section{Kontrolle der Beurteilenden}

Die Gerichtspräsidenten und Leiter der Staatsanwaltschaften unterliegen ebenfalls der Beurteilung durch den autonomen Justizrat. Diese Beurteilungen zeigen zweierlei: Erstens belegen sie die »Zwitter «-Natur (im Einzelfall als Richter oder als Staatsanwalt handelnd und gleichzeitig als Gerichtspräsident oder Leiter der Staatsanwaltschaft, verantwortlich für die Sicherstellung von Effizienz, aber mit besonderer Betonung ihrer Autonomie und Unabhängigkeit). Zweitens zeigen sie die Schwierigkeit, den »Output« der Rechtsprechung überhaupt zu bewerten.

In der Vergangenheit wurde die Kontrolle der Gerichtspräsidenten und der leitenden Staatsanwälte durch die Tatsache gegenstandslos, dass die jeweils zu Beurteilenden Garantien genossen, die ihre Versetzung verhinderten. Entscheidend war, dass ihre Tätigkeit überhaupt nur in außergewöhnlichen Fällen zur Beurteilung kamen, in denen sie dann aus dem Amt entfernt werden konnten oder sich Disziplinarmaßnahmen ergaben.

Der Gesetzgeber schritt 2006 schließlich ein und normierte, dass solche Posten zeitlich beschränkte Ernennungen waren. Damit ermöglichte er Beurteilungen, die nicht nur in außergewöhnlichen Fällen durchgeführt werden konnten, sondern regulär im Rahmen anstehender funktioneller Neubesetzungen. Es war eine bahnbrechende Entwicklung und die Auswirkungen müssen insgesamt erst noch abgewartet werden. Die Reformen werden auch die Rolle des CSM betreffen: Wenn die Leistung des Richters bzw. Staatsanwalts an standardisierten Vorgaben bemessen wird, führt das zwangsläufig dazu, dass eine tabellarische Beurteilung die Individualität des Richters/Staatsanwalts nivelliert. Dies wiederum wird der »politisch-institutionellen« Bewertung und damit den Verfahren der Entscheidungsfindung des CSM sogar noch größeres Gewicht verleihen.

Meines Erachtens ist es wahrscheinlich, dass es einen Anstoß-Effekt bezüglich der Legitimität des CSM und der Bedeutung des Justizrats geben wird: Es ist schwer vorstellbar, dass sie nicht Einflüssen lokaler Gremien ausgesetzt sind. Der CSM verdient indes Anerkennung für einen außergewöhnlichen Beweis an Effizienz, die auch die kühnsten Erwartungen übertroffen hat. In wenigen Monaten ist es ihm gelungen, den ersten Teil dieser Reform zu implementieren: Die gleichzeitige Ersetzung von mehr 
als 250 Gerichtspräsidenten, leitenden Staatsanwälten und sonstigen Leitungspositionen, bei denen die Zeitgrenze der Amtsdauer überschritten war. Noch wichtiger ist, dass dies durch vorwiegend einstimmige Resolutionen geschah.

Das Beurteilungssystem ist, wie wir gesehen haben, weit davon entfernt, als gefestigt zu gelten. Zusätzlich zu den Verzögerungen aus dem Justizministerium gibt es Schwächen, die mit dem System der Selbstverwaltung verbunden sind. Die Justizkultur zeigt noch kein derartig festes Profil, dass dadurch ein von allgemeiner Zustimmung getragenes Beurteilungssystem ermöglicht würde. Die Ablehnung aus der Richter- und Staatsanwaltschaft gegen Beurteilungen ist immer noch virulent. Es ist jedoch anzumerken, dass sich inzwischen ein allgemeines Bewusstsein der vitalen Bedeutung des Bewertungssystems für die Unabhängigkeit und Glaubwürdigkeit der Justiz herausgebildet hat, weil die unterschiedlichen, sich in diesem System begegnenden Verfassungsinteressen miteinander in Einklang zu bringen sind. Zudem darf nicht vergessen werden, dass die verfassungskonforme Beurteilung von Richtern und Staatsanwälten ein Mittel zur Sicherstellung der Qualität richterlicher und staatsanwaltlicher Tätigkeit ist und der Wahrung berechtigter Interessen der Bürger dient. Letzere haben ein Recht auf gut ausgebildete und unabhängige Richter und Staatsanwälte, die diesen Ansprüchen auch genügen.

\section{Berufliche Fortbildung}

Seit Jahren zeichnet sich ein bedeutender Aufgabenbereich des CSM ab: die berufliche Fortbildung. Der CSM hat sich für eine Fortbildung auf freiwilliger Basis ohne abschließende Prüfung ausgesprochen. Die Teilnahme an den Kursen wurde dennoch für die Zwecke der Beurteilungen als äußerst wichtig erachtet, da sie objektive Daten verfügbar macht, die auf spezifischen Einsatz und Interessensgebiete schließen lassen (Teilnahme an Studienkursen; Publikationen usw.). Die Fortbildungsprogramme wurden sowohl zentral (durch ein spezielles Komitee des CSM) als auch regional (mit dezentralisierter Fortbildung) geleitet. Die regionalen Kursleiter werden durch den CSM ernannt, der ihre Aktivitäten zusammen mit den Justizräten koordiniert. Das Konzept der Fortbildung hat sich in einem beeindruckenden Maß durchgesetzt. Jedes Jahr beteiligte sich etwa die Hälfte aller aktiven Richter und Staatsanwälte an den Fortbildungskursen. Einer von zwei Teilnehmern hat dabei sogar mehrere Kurse im selben Jahr absolviert. Führende Persönlichkeiten auf nationaler Ebene einschließlich zahlreicher Richter und Staatsanwälte haben an den Fortbildungsprogrammen teilgenommen. Das hohe Niveau der Tagungen hat weite Anerkennung gefunden und findet auch publizistischen Niederschlag. Die Fortbildungsprogramme nahmen einen großen Teil der Kapazitäten des CSM in Anspruch. Die Reform von 2006/2007 ermöglicht die Errichtung einer spezifischen Fortbildungsstätte als Reaktion auf die seit langem bestehende Nachfrage durch die Richtervereinigungen, die darin eine Möglichkeit sehen, Programme konkret zu gestalten.

Bei der Entscheidung über die praktische Durchführung hat die Exekutive mehr als einmal versucht, die Fortbildung der Kontrolle des CSM zu entziehen. Zugleich hat es Versuche gegeben, die Freiwilligkeit einzuschränken und ein Pflichttraining mit ab- 
schließenden Prüfungen einzuführen. Dies wäre jedoch mit einem hohen Risiko externer Einflussnahme gekoppelt. Die Freiheit von Beeinflussung (auch justizinterner) ist aber ein Grundpfeiler der Unabhängigkeit der Richter und Staatsanwälte. ${ }^{6}$

\section{Rahmenbedingungen für die Verhängung von Disziplinarmaßnahmen}

Die Verfassung gibt dem Obersten Justizrat die Kompetenz, Disziplinarmaßnahmen zu ergreifen. Die Disziplinarverfahren werden durch den Generalstaatsanwalt oder den Justizminister eingeleitet. Der Minister ist jedoch gezwungen, die Maßnahmen ausschließlich durch den Generalstaatsanwalt durchführen zu lassen, der für die Sicherstellung ihrer Verhältnismäßigkeit und Legalität verantwortlich ist. In der Vergangenheit wurden Disziplinarvergehen nicht typisiert. Die Disziplinarabteilung des CSM hat jedoch auf Grundlage der allgemeinen Prinzipien, die von der Legislative festgelegt wurden, Beispielskataloge von relevantem Verhalten gebildet, wie auch die Bedingungen für die Anwendung einer Sanktion typisiert (Zur Legitimität von nicht typisierten Verstößen siehe Verfassungsgerichts E Nr. 100/1981). Diese Interpretation diente als Eckpfeiler der Reform von 2006, in der Verstöße in allen Einzelheiten typisiert sind. Die Auffassung ist allgemein verbreitet, dass die Disziplinarjustiz des CSM zu milde und ziemlich ineffektiv sei. Die politische Debatte spricht von »Hausjustiz«. Ständige Wiederholungen solcher Ansichten haben den Effekt, sie als wahr erscheinen zu lassen, auch wenn sie es nicht sind. Empirische Daten belegen deutlich, dass die Disziplinarjustiz des CSM rigoroser ist als die irgendeines vergleichbaren Systems.

Die Zahlen sprechen deutlich für die Effizienz dieser Institution: Während der letzten zehn Jahre wurden ungefähr 900 Richter bzw. Staatsanwälte Disziplinarverfahren unterworfen. Dies sind ungefähr $10 \%$ aller Richter und Staatsanwälte, und von diesen wurden ungefähr $40 \%$ entweder sanktioniert oder zogen es vor, den Dienst zu quittieren, bevor eine Entscheidung getroffen wurde. Die Schwierigkeiten, die in der Vergangenheit bei der effektiven Bewertung richterlicher bzw. staatsanwaltlicher Arbeit aufgetreten sind, haben zu einer enormen Ausdehnung von Disziplinarverfahren geführt: Eine Disziplinarsanktion führte zum gleichen Ergebnis wie eine negative Bewertung bei der Beurteilung. Es bleibt zu hoffen, dass ein System fortlaufender Überwachung, welches die rechtsprechenden Pflichten beobachtet, und dabei auch effizient ist, in der Lage sein wird, in Zukunft die Wiederholung von Zuständen dieser Art zu

6 In diesem Kontext hat der CSM kürzlich die Aufmerksamkeit auf Grundsätze gelenkt, die auch im Konflikt mit Art. 105 der Verfassung standen: »Angesichts dieser Funktionen (scil. Selbstverwaltung) wird es als unannehmbar betrachtet, dass Richter/Staatsanwälte entweder durch ein unmittelbares Staatsorgan, durch äußere Gewalten oder die Gewalt der Justiz selbst angewiesen werden, bei der Interpretation des Gesetzes bestimmte Sichtweisen oder Standpunkte einzunehmen. Folglich hat der Richter/Staatsanwalt, der ohne Unterstützung von außen allein dem Gesetz verantwortlich ist, das er auslegen muss, ein größeres Bedürfnis nach fortlaufender Schulung auf höchstem Niveau als irgendein anderer Staatsbediensteter. Ihm müssen die Ressourcen zustehen, die notwendig sind, das Gesetz anzuwenden, wofür er die volle Verantwortung übernimmt « (Bericht aus dem Jahr 1994 an das Parlament zur Frage der professionellen Schulung für Richter/Staatsanwälte). 
verhindern. Es werden ständig Vorschläge gemacht, die Disziplinargewalt aus dem Zuständigkeitsbereich des CSM herauszunehmen. Ich meine, dass dies ein Fehler wäre. Erstens hat der CSM sich bis jetzt als die beste und strengste disziplinarrechtliche Körperschaft erwiesen, vielleicht in zu großem Maße. Zweitens sollte die justizeigene Natur der Disziplinargewalt eher positiv zu bewerten sein: Sie deutet in keiner Weise auf eine Art von Justiz hin, die als gezähmt oder selbstbezogen beschrieben werden könnte. In der Tat ist genau das Gegenteil der Fall: Nur durch die Aufrechterhaltung eines ethischen Verhaltenskodex, der nicht nur Disziplinarvergehen betrifft, und durch das Bestehen auf professionelle Standards kann Disziplinargewalt ein positiver Faktor für die Justiz sein. Die Erfahrung mit dem Obersten Justizrat zeigt dies deutlich. 